\title{
Micromagnetic modeling and imaging of vortex|meron structures in an oxide|metal heterostructure
}

\author{
P. G. Radaelli, ${ }^{1}$ J. Radaelli, ${ }^{1,2}$ N. Waterfield-Price $\odot,{ }^{1}$ and R. D. Johnson ${ }^{1,3}$ \\ ${ }^{1}$ Clarendon Laboratory, Department of Physics, University of Oxford, Parks Road, Oxford OX1 3PU, United Kingdom \\ ${ }^{2}$ Department of Physics, University of Durham, Lower Mountjoy, South Road, Durham DH1 3LE, United Kingdom \\ ${ }^{3}$ Department of Physics and Astronomy, University College London, London WC1E 6BT, United Kingdom
}

(Received 17 December 2019; revised manuscript received 24 February 2020; accepted 26 February 2020; published 14 April 2020)

\begin{abstract}
Using micromagnetic simulations, we have modeled the formation of imprinted merons and antimerons in cobalt overlayers of different thickness $(1-8 \mathrm{~nm})$, stabilized by interfacial exchange with antiferromagnetic vortices in $\alpha-\mathrm{Fe}_{2} \mathrm{O}_{3}$. Structures similar to those observed experimentally could be obtained with reasonable exchange parameters, also in the presence of surface roughness. We produce simulated meron and antimeron images by magnetic force microscopy and nitrogen-vacancy center microscopy, and established signatures of these topological structures in different experimental configurations.
\end{abstract}

DOI: 10.1103/PhysRevB.101.144420

\section{INTRODUCTION}

"Oxide electronics" aims at combining the multifunctional properties of transition-metal oxides with more traditional spintronic approaches and represents one of the most promising pathways to post-complementary metal-oxide semiconductor computing [1]. One approach is to exploit the rich real-space topological properties of oxide domains to create structures such as vortices and skyrmions, which could be "imprinted" onto ferromagnetic (FM) read-out overlayers. Heterostructures of this kind, particularly those built with rare-earth-free materials, could be used as high-density, nonvolatile memories with a high degree of thermal stability.

Skyrmions are the best known example of magnetic topological object, and have received an enormous amount of attention (see for example [2,3] for recent reviews). Magnetic merons and antimerons (essentially flat vortices and antivortices with an out-of-plane core) have been known to exist as closure domains in magnetic nanodots since the early 2000s [4] and have more recently been observed in extended systems, either as intermediate stages of skyrmion array formation in chiral magnets [5] or as light-induced metastable magnetic textures in the absence of in-built chirality [6]. Both skyrmions and merons can be thought of as projections onto a tangent plane of a vector field defined on the surface of a sphere, the projection point being either the center of the sphere (meron) or the opposite pole (skyrmion) [3]. As such, these objects can be characterized by a topological charge or winding number, $w$. The magnitude $|w|$ counts how many times the vector field wraps around the sphere or half sphere, while the sign of $w$ defines the direction of wrapping. The topological charge, which can be calculated as a surface integral of the projected field [3], is a positive (negative) integer for skyrmions (antiskyrmions) and a positive (negative) halfinteger for merons (anti-merons) [3]. One important property of the topological charge is that, being an integer or half integer, it must change discontinuously, and is therefore left invariant by smoothly varying rotations in spin space. In other words, in order to alter the topological charge of a given object, one must introduce a singularity in the local field. Since this tends to be associated with a high energy cost, these objects are often said to be "topologically protected." In real magnetic systems, topological objects do not enjoy an absolute protection (for example, they can annihilate with their own antiparticles) but are often very stable against thermal fluctuations.

Using a combination of $\mathrm{x}$-ray magnetic linear and circular dichroism photoelectron emission microscopy (XMLD/XMCD-PEEM), we have recently demonstrated that antiferromagnetic (AFM) planar vortices and antivortices exist in $\alpha-\mathrm{Fe}_{2} \mathrm{O}_{3}$, and that these structures are "imprinted" as FM vortices onto a 1-nm soft Co overlayer [7]. Although our XMCD-PEEM measurements were not conclusive due to limitations in spatial resolution, they were consistent with an out-of-plane component of the Co spins, which would make the Co structures merons and antimerons [8] rather than planar vortices. If corroborated, the observation of merons and antimerons would be extremely important, since the out-of-plane spin component would represent a convenient two-bit state, which, similar to skyrmions, is to a large extent topologically protected [9]. Another conclusion of our experimental work was that spins in $\mathrm{Co}$ are co-aligned with the $\alpha-\mathrm{Fe}_{2} \mathrm{O}_{3}$ AFM spins, indicating that the interaction responsible for the vortex|meron coupling is akin to exchange bias [10] (hereafter, we refer to this as "exchange-bias interaction") rather than the $90^{\circ}$ interaction observed in other systems [11]. This raises another important question: Since AFM spins in $\alpha-\mathrm{Fe}_{2} \mathrm{O}_{3}$ have opposite directions for adjacent terminations, how can Co merons and antimerons be stable in the presence of surface roughness?

In this paper, we model the combined vortex|meron and antivortex|antimeron structures we have observed in $\alpha-\mathrm{Fe}_{2} \mathrm{O}_{3} \mid \mathrm{Co}$ using micromagnetic simulations. We demonstrate that Co merons or antimerons are stabilized by an underlying $\alpha-\mathrm{Fe}_{2} \mathrm{O}_{3}$ vortex or antivortex due to a competition between exchange stiffness, exchange-bias interaction, and 
magnetostatic interaction. Correspondingly, the scale of the Co features is governed by the two exchange lengths, $L_{e x . b}$, which accounts for the field induced by the interface, and the usual magnetostatic length, $L_{m s}$. We also determine the scaling of the meron core with the exchange parameters and film thickness, and establish that (anti)vortex|meron structures are stable for rough interfaces, provided that the characteristic scale of the roughness is less than the exchange lengths. Finally, we construct simulated scanning probe microscopy (SPM) images of the Co features using both magnetic force microscopy (MFM) and nitrogen-vacancy (NV) center microscopy, and identified characteristic signatures that could be detected in the experiments. Although our calculations and simulations are carried out for the $\alpha-\mathrm{Fe}_{2} \mathrm{O}_{3} \mid \mathrm{Co}$ system, our methodology is of general validity for exchange-coupled topological structures, and could be applied to a variety of systems of interest for oxide electronics and spintronics. Light-induced metastable vortices [6] are also described by our analysis as a limiting case in which $L_{e x . b}=0$.

The paper is organized as follows: In Sec. II we make dimensional considerations based on the key physical parameters and discuss a simple analytical model of a vortex|meron structure. In Sec. III, we discuss our approach to micromagnetic simulations, in particular, providing a conversion between the atomic-scale and micromagnetic parameters, simulating surface roughness and constructing simulated SPM images. Section IV contains the main results concerning meron stability (also in the presence of surface roughness), and the scaling of the meron and antimeron cores, as well as our simulated MFM and NV center microscopy images, and is followed by a short conclusion.

\section{THEORY}

\section{A. Brief description of the physical system}

Our goal was to model the coupled vortex|meron structures observed by XMLD/XMCD-PEEM at room temperature (RT) (see Ref. [7]). In this experiment, the physical system consisted of a 10-nm epitaxial [001] $\alpha-\mathrm{Fe}_{2} \mathrm{O}_{3}$ film grown on sapphire $\left(\mathrm{Al}_{2} \mathrm{O}_{3}\right)$, with 1-nm FM Co capping layer grown at RT by dc sputtering. The RT magnetic structure of $\alpha-\mathrm{Fe}_{2} \mathrm{O}_{3}$ consists of collinear AFM layers (we ignore the small in-plane spin canting), stacked along the [001] direction in a repeated pattern ".+--+ ." All spins are perpendicular to the stacking direction and are aligned along one of the symmetry-equivalent $\{100\}$ directions, so that six equivalent domains are possible. A network of AFM vortices and antivortices is experimentally observed by XMLD-PEEM, where six domains meet at a single point. Very similar topological structures are also observed by XMCD-PEEM in the Co overlayer exactly on top of the $\alpha-\mathrm{Fe}_{2} \mathrm{O}_{3}$ structures and having the same vorticity (vortex or antivortex character and direction of rotation). The XMCD-PEEM vector-map intensity (proportional to the in-plane projection of the magnetic moment) shows a pronounced dip near the FM vortex cores, suggesting the presence of an out-of-plane $(z)$ component, which is characteristic of merons. Since the size of the observed meron "cores" (i.e., the region where a sizable $z$ component exists) was comparable to the typical x-ray PEEM (X-PEEM) resolution of 20-50 nm, it was not possible to establish the actual core size with any confidence.

\section{B. Feature sizes: Dimensional considerations}

Consistent with our experimental findings, we assume that Co experiences a bulk FM self-interaction, described by an exchange stiffness $A_{e x}^{\text {Co-Co }}$ (in $\mathrm{J} \mathrm{m}^{-1}$ ), as well as a surface interaction with $\alpha-\mathrm{Fe}_{2} \mathrm{O}_{3}$ described by an "exchange-bias" constant $K_{e x . b}^{\mathrm{Fe}-\mathrm{Co}}$, having dimensions $\mathrm{J} \mathrm{m}^{-2}$ (we drop the unambiguous superscripts in the remainder).

In our simulations, we assume that the domain structures in $\alpha-\mathrm{Fe}_{2} \mathrm{O}_{3}$ are rigid (i.e., not affected by the presence of the overlayer), that they have much narrower domain walls than those in $\mathrm{Co}$, and that all the $\alpha-\mathrm{Fe}_{2} \mathrm{O}_{3}$ spins lie in plane. At present, there is no experimental verification for these assumptions, which may not in fact be entirely correct. In fact, since the energies of the in-plane and out-of-plane spin orientations are finely balanced, $\alpha-\mathrm{Fe}_{2} \mathrm{O}_{3}$ could even support AFM merons with an out-of-plane core [12,13], while "reverse imprint" of a FM overlayer on an AFM layer has been previously discussed for other materials [14]. However, such a coupled problem would be intractable at the micromagnetic level, while the effect of a finite width of the $\alpha-\mathrm{Fe}_{2} \mathrm{O}_{3}$ domain walls can be easily included in our models, should any solid experimental evidence emerge. We therefore believe that our assumptions are justified, in that they provide a simplified but useful model of the relevant physics.

Initially, we also assume that $\alpha-\mathrm{Fe}_{2} \mathrm{O}_{3}$ has a FM termination with no roughness (we relax this assumption later). With these assumptions, the other key physical parameter in the problem is the thickness $d$ of the Co film. From these parameters, one can construct a length,

$$
L_{e x . b}=\sqrt{\frac{A_{e x} d}{K_{e x . b}}} .
$$

There is also a second length scale in Co, the "conventional" magnetostatic length [15], unrelated to the presence of $\alpha-\mathrm{Fe}_{2} \mathrm{O}_{3}$ and given by

$$
L_{m s}=\sqrt{\frac{2 A_{e x}}{\mu_{0} M^{2}}},
$$

where $M$ is the Co magnetization. From this simple analysis one should conclude that the size of any magnetic feature in Co should be determined by the competition between two lengths, $L_{e x . b}$ and $L_{m s}$, which control the "surface" and "bulk" physics of the problem, respectively. Moreover, when one of the lengths is much larger than the other, Co features should roughly scale with the smaller of the two lengths.

One could test this prediction by calculating, for example, the shape and width of a Néel domain wall induced by the presence of a sharp antiphase boundary in the underlying AFM material. Problems of this kind have been discussed at the micromagnetic level since the 1960s [16] and involve differential equations imposing zero torque on each magnetization element [17]. Although finding full analytical solutions is beyond the scope of this paper (which focuses on numerical solutions of these equations when $L_{e x . b}$ and $L_{m s}$ are comparable), in Appendixes $\mathrm{B}$ and $\mathrm{C}$ we present simple solutions 
of the linear domain wall and of the vortex|meron problem, respectively, assuming the shape of these structures to be a known function. The linear Néel domain wall problem with uniform spin rotation is very similar to the well-known calculation of the width of a Bloch domain wall in the presence of magnetocrystalline anisotropy [15], and leads to a very similar result:

$$
W_{N}=\pi\left(1-\frac{2}{\pi}\right)^{-1 / 2} L_{e x . b} \approx 5.21 L_{e x . b}
$$

where $L_{\text {ex.b }}$ replaces the usual magnetocrystalline anisotropy exchange length [15]. Equation (3) is strictly valid in the limit $\frac{d}{L_{e x .}} \ll 1$. This is appropriate throughout most of the range we consider, since we typically set $d=1 \mathrm{~nm}$ in agreement with Ref. [7], while typical domain wall widths in our simulations are $\geqslant 4 \mathrm{~nm}$. Significant departures from this approximation are considered in Sec. IV B.

\section{Analytical merons}

To reinforce the results from the previous section, we perform an analytical calculation of a "model" meron (or antimeron) in Co, stabilized by the presence of a planar vortex (or antivortex) in the adjacent AFM oxide, assuming very simple functional forms for the $z$ component of the magnetization. We demonstrate that the characteristic size of the meron "core" is indeed proportional to $L_{e x . b}$. In this calculation, we disregard the effect of magnetostatic energy (included in our micromagnetic model; see below), so the calculation is exactly identical for vortex|meron and antivortex|antimeron structures.

The general expression for the normalized meron magnetization is

$m_{x}=-\sin \psi \sin \phi, \quad m_{y}=\sin \psi \cos \phi, \quad m_{z}=\cos \psi$,

where $\phi$ is the polar angle and

$\tan \psi=\mathcal{F}\left(\frac{r}{R}\right), \quad \cos \psi=\frac{1}{\sqrt{\mathcal{F}^{2}+1}}, \quad \sin \psi=\frac{\mathcal{F}}{\sqrt{\mathcal{F}^{2}+1}}$.

Here, $\mathcal{F}(x)$ is a continuous function with $\lim _{x \rightarrow 0} \mathcal{F}(x)=0$ and $\lim _{x \rightarrow \infty} \mathcal{F}(x)=\infty$, and $R$ is a characteristic scale. In Appendix $\mathrm{C}$ we provide calculations for a number of simple cases, including the "projective" meron $(\mathcal{F}(x)=x)$, which can be obtained by projecting a "hairy" sphere of radius $R$ from its center onto a tangent plane [18], and the more general case in which $\mathcal{F}(x)$ is a polynomial. In order to provide a direct comparison with the linear domain wall, we also discuss the case of the "linear meron," where the magnetic moment is entirely in plane outside a radius $R$, while inside this radius it rotates uniformly towards the center of the meron, where it is aligned along $z$. As shown in Appendix $\mathrm{C}$, the projective meron case is unstable, due to the logarithmic energy cost owing to the swirling spins at large distances, while in all other cases the width of the meron core scales with the exchangebias length $L_{\text {ex.b }}$ [see also Eq. (C16)]:

$$
W_{\text {core }}=2 \kappa \sqrt{\frac{A_{e x} d}{K_{e x . b}}}=2 \kappa L_{e x . b}
$$

with $\kappa \approx 1$.

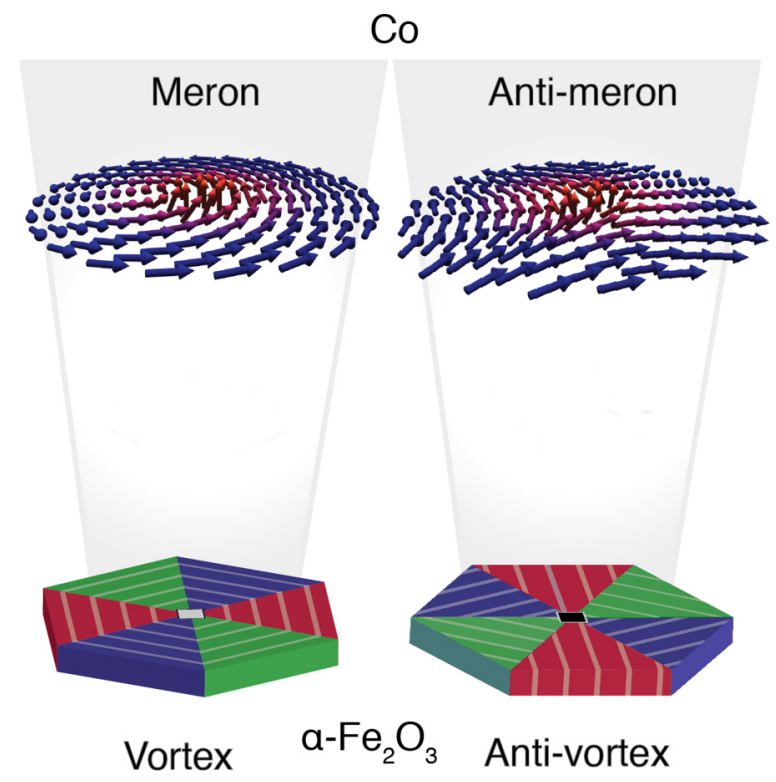

FIG. 1. Overview of the coupled $\alpha-\mathrm{Fe}_{2} \mathrm{O}_{3} \mid \mathrm{Co}$ system that was simulated in this section. The bottom layer is $\alpha-\mathrm{Fe}_{2} \mathrm{O}_{3}$ and the figure shows a vortex (bottom left) and an antivortex (bottom right). Lightcolored lines indicating the direction of the staggered magnetization. Only the top termination of $\alpha-\mathrm{Fe}_{2} \mathrm{O}_{3}$ was included as a fixed layer in the simulations (see Fig. 2, left panel). Spins in the top Co layer were set to a random orientation prior to the start of the simulation, and develop meron and antimeron structures at the end of the simulation, as shown in the top panels.

\section{MICROMAGNETIC MODELING}

\section{A. Micromagnetic modeling in OOMMF}

Micromagnetic simulations were performed using the program OOMMF [19]. No periodic boundary conditions were employed and the AFM layers were kept fixed throughout the simulations in all cases. A general overview of the system we simulated is provided in Fig. 1. Uniform $\alpha-\mathrm{Fe}_{2} \mathrm{O}_{3}$ termination layers were described as having magnetization of constant magnitude, which rotates counterclockwise (for vortices) or clockwise (for antivortices) when moving on a counterclockwise path around the center. The total simulated area was $200 \times 200 \mathrm{~nm}^{2}$ and the discretization cell sizes were $D_{x y}=2 \mathrm{~nm}$ and $D_{z}=1 \mathrm{~nm}$, respectively, with the $\alpha-\mathrm{Fe}_{2} \mathrm{O}_{3}$ layer being one cell thick. We performed simulations both with uniformly rotating magnetization and also with constant magnetization within six equal wedges, which reproduce the experimental images of AFM vortices and antivortices [7] (Fig. 2, left-hand panel). The meron structures in the two cases are extremely similar, although the sharp AFM boundaries associated with the wedges introduce Néel domain walls (see below). To model the effect of surface roughness, an additional set of simulations was performed over $100 \times 100 \mathrm{~nm}^{2}$ with $D_{x y}=0.5 \mathrm{~nm}$ in-plane discretization, with the $\alpha-\mathrm{Fe}_{2} \mathrm{O}_{3}$ magnetization being reversed within circular islands arranged on a regular grid (Fig. 2, right-hand panel).

Co layers of different thickness (1-8 nm) were placed in direct contact with the $\alpha-\mathrm{Fe}_{2} \mathrm{O}_{3}$ layer and interacting with 

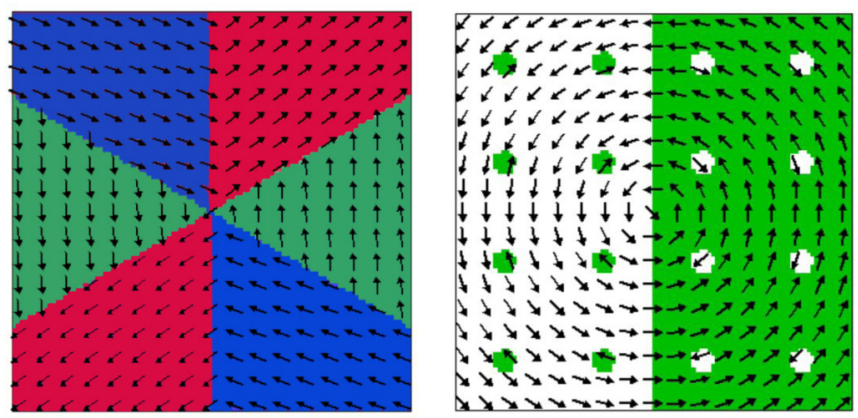

FIG. 2. Termination layers of $\mathrm{AFM} \alpha-\mathrm{Fe}_{2} \mathrm{O}_{3}$. Left: Antivortex in $\alpha-\mathrm{Fe}_{2} \mathrm{O}_{3}$ with six distinct wedges (colors or grayscale), as observed in Ref. [7]. The simulated area is $200 \times 200 \mathrm{~nm}^{2}$. Right: Vortex with uniformly rotating magnetization and circular regions of magnetization reversal, introduced to simulate surface roughness. The diameter of the circular regions (emphasized by the shading) is $6 \mathrm{~nm}$, while the simulated area is $100 \times 100 \mathrm{~nm}^{2}$. Colors or shading emphasize the moment directions.

it through an exchange stiffness $A_{e x}^{\mathrm{Fe}-\mathrm{Co}}$ (which is not known a priori; see below and Appendix A for a full discussion), so a series of simulations was performed spanning a wide range of $L_{e x . b}$. For $A_{e x}^{\text {Co-Co }}$, we have used the literature value of $18 \mathrm{pJ} \mathrm{m}^{-1}$ [15]. The Co magnetization is assumed to be $1.4 \times 10^{6} \mathrm{~J} \mathrm{~T}^{-1}$, yielding a magnetostatic exchange length $L_{m s}=\sqrt{2 A_{e x} / \mu_{0} M^{2}}=3.8 \mathrm{~nm}$ [15]. The magnetization in each cell was initially set at a random orientation, and it was then allowed to evolve according to the Landau-LifshitzGilbert equation [20] until a stable configuration was attained. Since here we are not interested in magnetization dynamics, the dimensionless damping factor $\alpha$ should not influence the outcome; in our simulations, $\alpha$ was set to 0.5 - a value that was empirically found to yield good convergence properties of the model. The simulation time step was adjusted by the program in the range $1-100 \mathrm{ps}$, while the convergence criterion was $5 \mathrm{~ns}^{-1}$. Unless $A_{e x}^{\mathrm{Fe}-\mathrm{Co}}$ was set to a very small value, the Co magnetization always formed a meron or antimeron, with the same vorticity as the underlying vortex or antivortex in $\alpha-\mathrm{Fe}_{2} \mathrm{O}_{3}$ while the core magnetization was randomly up or down in each simulation run.

Although strictly a technical issue, the implementation of exchange bias in our micromagnetic simulations deserves a separate remark, since in OOMMF it is not possible to introduce the equivalent of $K_{\text {ex.b }}$ directly. Instead, the effect of exchange bias can be reproduced by employing an exchange stiffness $A_{e x}^{\mathrm{Fe}-\mathrm{Co}}$, which acts only on the interface cells between $\alpha-\mathrm{Fe}_{2} \mathrm{O}_{3}$ and Co. The only caveat is that $A_{e x}^{\mathrm{Fe}-\mathrm{Co}}$ is not a physical parameter, since it depends on the size of the discretization cell $D_{z}$ along the $z$ direction, as discussed at length in Appendix A. The scaling $K_{e x . b}=2 A_{e x}^{\mathrm{Fe}-\mathrm{Co}} / D_{z}$ between $A_{e x}^{\mathrm{Fe}-\mathrm{Co}}$ and the physical parameter $K_{e x . b}$, derived in Appendix A, was verified in a series of simulations with different $D_{z}$.

\section{B. Modeling MFM and NV center microscopy images}

Simulated MFM and diamond NV center microscopy images were produced from meron and antimeron structures obtained by setting $L_{e x . b}=L_{m s}$ as a representative value.
For MFM, we employed the phase-shift method, whereby the image is generated based on the shift in phase between the drive and the cantilever, which is driven close to resonance [21]. The phase shift is given by the formula

$$
\Delta \Phi=-\frac{Q}{k} \frac{\partial F_{z}}{\partial z},
$$

where

$$
\mathbf{F}=\nabla(\boldsymbol{\mu} \cdot \mathbf{H})
$$

is the force on the cantilever tip due to the stray magnetic field $\mathbf{H}$, and $\boldsymbol{\mu}$ is the magnetic moment of the tip. The derivative of the force was calculated numerically and averaged over a number of "voxels" comprising the shape of the tip. The magnetic moment of the tip was kept constant at $|\boldsymbol{\mu}|=1.2 \times$ $10^{-19} \mathrm{~J} \mathrm{~T}^{-1}$, while different tip sizes and shapes were tested. The cantilever spring constant in Eq. (7) was $k=2.8 \mathrm{~N} \mathrm{~m}^{-1}$, while the quality factor $Q$ was set at 100 , which is much less than the "bare" cantilever $Q$ but is realistic for roomtemperature measurements in the presence of a water film.

For diamond NV center microscopy, a first set of images was produced without bias magnetic field, assuming that the signal is proportional to the magnitude of the projection of the stray magnetic field along the direction of the defect, which was aligned with the [111] crystallographic direction of the diamond [22,23]. The [001] and [110] crystallographic directions of the diamond were aligned along the $z$ and $x$ axes, respectively. A second set of images was produced with a bias field of $\sim 110 \mathrm{mT}$ along the $x$ direction, such that the projection of the stray plus bias magnetic field along the defect never changes sign. This field should be considered an upper limit of what it is possible to apply experimentally, since a field of this magnitude on the surface of the sample is likely to cause meron annihilation [7].

\section{RESULTS}

\section{A. Meron and antimeron formation and feature size}

Figure 3 shows typical "converged" Co spin configurations for a meron [Fig. 3(a)] and an antimeron [Fig. 3(b)] stabilized by an AFM vortex or antivortex, similar to that in Fig. 2 (left-hand panel), using exchange lengths $L_{e x . b}=L_{m s}=L_{e x . b}$ $=3.8 \mathrm{~nm}$. Figure 3(c) shows the $z$ component of the magnetization plotted along a line cutting through the meron core, while Fig. 3(d) shows the component of the magnetization perpendicular to the underlying AFM spins, plotted along a line cutting through a Néel domain wall [lines shown in Fig. 3(a)] [24]. At the center of the meron or antimeron, the magnetization is completely aligned along the $z$ axis. For the meron, $M_{z}$ is nonzero only near the core, while for the antimeron there is a sizable $M_{z}$ component along the two diagonal lines where the in-plane magnetization is along the radial direction. Interestingly, the full width at half maximum (FWHM) of the meron core $(6 \mathrm{~nm})$ is smaller than that of the Néel domain wall $(7.7 \mathrm{~nm}$ ) (this discrepancy is qualitatively consistent with the analytical results in Appendices B and C). 

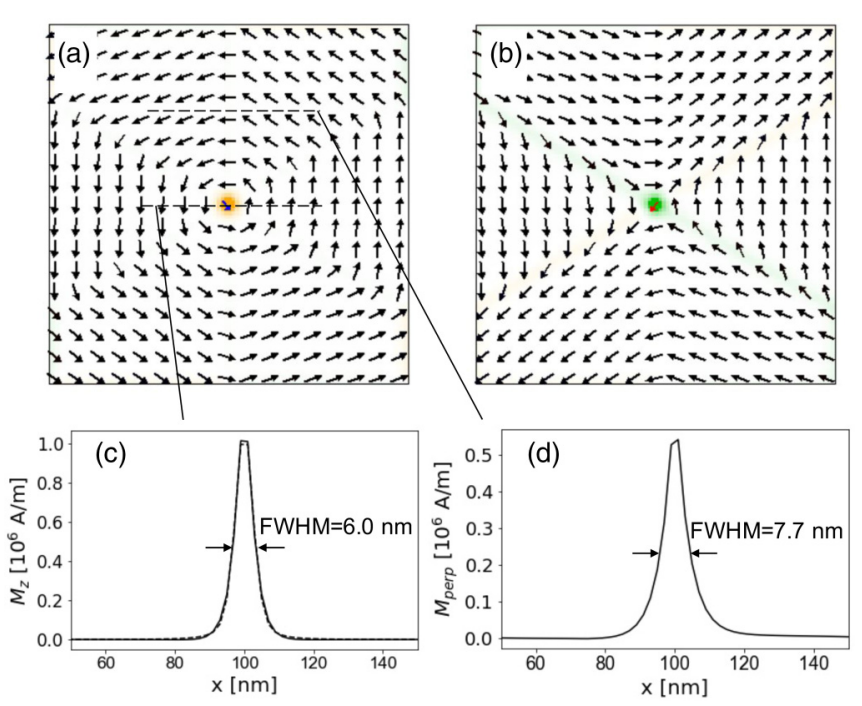

FIG. 3. Top: $200 \times 200 \mathrm{~nm}^{2}$ OOMMF simulations of (a) a meron and (b) an antimeron in a $1 \mathrm{~nm}$ Co film, with an exchange-bias field from a hexagonal vortex or antivortex in $\alpha-\mathrm{Fe}_{2} \mathrm{O}_{3}$. The exchange-bias length for the simulations was set at $L_{e x . b}=3.8 \mathrm{~nm}$. Away from the (anti)meron core, Co spins and Fe spins are parallel. The in-plane spin component is indicated by the arrows, while the out-of-plane $(z)$ components are in shaded color (grayscale). Bottom: Profiles of the magnitudes of Co spin component (c) along $z$ through the meron core and (d) orthogonal to the Fe spins through a Néel domain boundary.

\section{B. Meron and antimeron core scaling}

Having established the basic procedure to produce micromagnetic simulations of merons and antimerons, we proceeded to generate a series of structures with different values of $L_{e x . b}$, while keeping $L_{m s}$ at the literature value of $3.8 \mathrm{~nm}$. In establishing an appropriate range for $L_{e x . b}$, one should consider that, for an ideal system, the exchange stiffness and interface energies are related to the microscopic exchange constants $J$ by the following equations (see Ref. [25]):

$$
A_{e x}^{\mathrm{Co}-\mathrm{Co}}=c_{1} \frac{J^{\mathrm{Co}-\mathrm{Co}} S_{\mathrm{Co}}^{2}}{a_{n n}}, \quad K_{e x . b}^{\mathrm{Co}-\mathrm{Fe}}=c_{2} \frac{J^{\mathrm{Co}-\mathrm{Fe}} S_{\mathrm{Co}} S_{\mathrm{Fe}}}{a_{n n}^{2}},
$$

where $a_{n n}$ is the atomic nearest-neighbor distance, $S_{\mathrm{Fe}}$ and $S_{\text {Co }}$ are the cobalt and iron spins, while $c_{1}$ and $c_{2}$ are small numbers that depend on the coordination and $c_{1}>c_{2}$. In the approximation of equal exchange constants and spins, for an ideal system one would have

$$
L_{e x . b} \approx \sqrt{\frac{c_{1}}{c_{2}} d a_{n n}}
$$

so for $d=1 \mathrm{~nm}$ it is reasonable to take 1-2 $\mathrm{nm}$ as the lower bound for $L_{e x . b}$. In a real system, one would expect that $K^{\mathrm{Co}-\mathrm{Fe}}$ should be significantly weakened by surface roughness, so we tested much larger values of $L_{e x . b}$ (up to $35 \mathrm{~nm}$ ), up until the point where merons and antimerons ceased to be stable.

Once the models had converged, the meron and antimeron cores were fitted by two-dimensional pseudo-Voigt functions, which enabled the FWHM to be extracted systematically. The results of these fits are summarised in Fig. 4. For very strong exchange-bias interactions (small values of $L_{e x . b}$ ), interface physics is dominant, and the core size is proportional to $L_{e x . b}$,

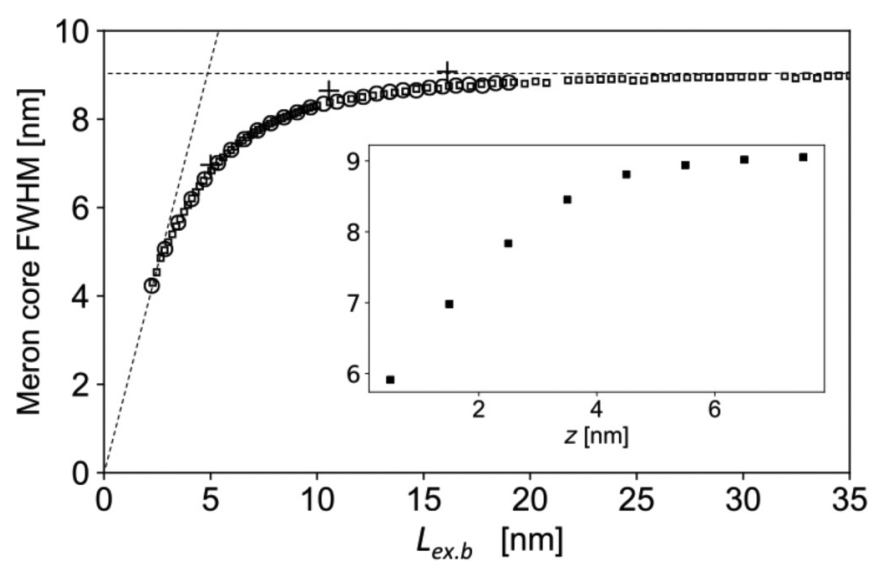

FIG. 4. Full width at half maximum of the meron core as a function of the exchange-bias length $L_{\text {ex.b }}$, as defined in Eq. (1). The anisotropy length $L_{m s}$ was set at $3.8 \mathrm{~nm}$ for all data points. Open squares and open circles correspond to Co film thicknesses $d$ of $1 \mathrm{~nm}$ and $2 \mathrm{~nm}$, respectively, while crosses are for antimerons with $d=1 \mathrm{~nm}$. The dashed lines correspond to $\sim 2.37 L_{m s}$ (horizontal) and $1.67 L_{\text {ex.b }}$ (diagonal). Inset: Meron core FWHM versus distance $z$ from the interface for an 8-nm Co film with $L_{\text {ex.b }}=3.8 \mathrm{~nm}$.

consistent with our analytical calculations (Appendix C). In fact, the proportionality constant extracted from the initial slope of the plot $(\approx 1.67)$ is rather close to the analytical value of 1.5 [Eq. (C17)]. For larger values of $L_{e x . b}$, the core size in increasingly dominated by "bulk" physics, and eventually saturates at $\approx 2.37 L_{m s}$. Meron and antimeron core sizes are almost identical for small $L_{e x . b}$, as one would expect, but antimeron cores are slightly bigger for larger $L_{e x . b}$, consistent with the fact that antivortices have very unfavorable magnetostatic energies. Compared to merons, antimerons ultimately become unstable for smaller values of $L_{e x . b}$.

For small film thicknesses (1-2 nm), the magnetization is essentially independent of $z$ and the effect of the thickness $d$ can be included in the definition of $L_{e x . b}$ given by Eq. (1). For thicker films, this ceases to be true, as shown in the inset of Fig. 4, which demonstrates the transition from "surface" to "bulk" physics within the same film. In the example shown $\left(L_{e x . b}=3.8 \mathrm{~nm}\right)$, the meron core is compact in the portion of the film closer to $\alpha-\mathrm{Fe}_{2} \mathrm{O}_{3}$ but flares out as $z$ increases, until it saturates to the bulk value of $\sim 2.37 \mathrm{~L}_{\mathrm{ms}}$.

One conclusion of this section is that the meron and antimeron core size in Co never exceeds $\sim 9 \mathrm{~nm}$ regardless of the strength of the interface interaction and the film thickness. This has very important implications for the possibility of creating dense meron and antimeron networks (see discussion at the end of the paper). A second observation is that, based on our simulations, there is likely to be a difference in the pinning strength required to keep merons and antimerons pinned to $\alpha-\mathrm{Fe}_{2} \mathrm{O}_{3}$, due to their different magnetostatic energy. This feature is amenable to be exploited for applications, for example, to "unpin" one type of particle selectively.

\section{Modeling surface roughness}

As previously mentioned (see Sec. IV B), our initial assumption of a uniform FM termination for $\alpha-\mathrm{Fe}_{2} \mathrm{O}_{3}$ cannot 


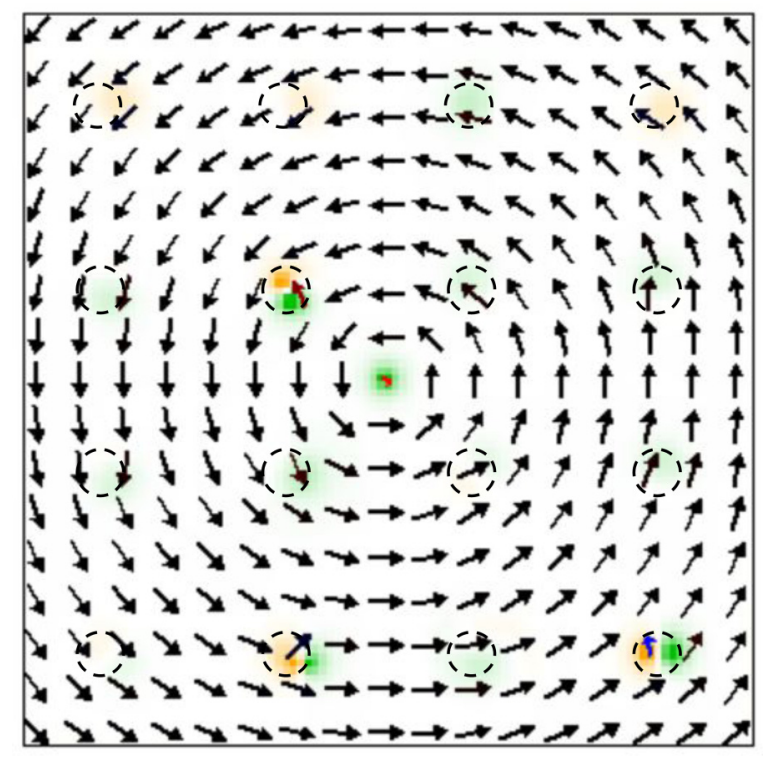

FIG. 5. Effect of $\alpha-\mathrm{Fe}_{2} \mathrm{O}_{3}$ roughness on the meron structure. 200 $\times 200 \mathrm{~nm}^{2}$ OOMMF simulation of a meron in a 1-nm-thick Co layer, stabilized by a "rough" $\alpha-\mathrm{Fe}_{2} \mathrm{O}_{3}$ interface. Roughness is simulated by introducing disklike areas of AFM spin reversal, with a diameter of $6 \mathrm{~nm}$ (dotted lines). The exchange lengths were set at $L_{e x . b}=$ $L_{m . s}=3.8 \mathrm{~nm}$. Color intensity is proportional to the out-of-plane component of the Co moments.

be realistic, since in all but the most perfect epitaxial films there is always a degree of surface roughness. One may even question whether merons and antimerons can be stabilized in the presence of a rough $\alpha-\mathrm{Fe}_{2} \mathrm{O}_{3}$ interface, since the sign of the magnetization in the layer in direct contact with Co changes sign in different termination layers. Intuitively, one would expect the lateral scale of the termination terraces to be an important parameter: features in Co cannot be smaller than one to two times the relevant exchange length, so the effect of fine-grained roughness should be to weaken the dominant exchange-bias interaction without altering the topology of the Co features. This intuition is confirmed by our micromagnetic models (shown in Fig. 5), in which surface roughness is simulated by regions of $\alpha-\mathrm{Fe}_{2} \mathrm{O}_{3}$ spin inversion in the shape of circular "terraces" of $6 \mathrm{~nm}$ diameter. In order to prevent the roughness-related features from being washed out by finitescale effects, these simulations were performed on smaller discretization cells $(0.5 \mathrm{~nm})$. As evident from Fig. 5, the shape of the meron structure in Co is largely unaffected by our model roughness. The main effect of the terraces is to introduce a small local distortion and a nonzero $z$ component of the magnetization - a very reasonable result, since this lowers the exchange-bias energy at the terrace site.

\section{MFM imaging and NV center imaging}

Figure 6 shows simulated MFM images of a meron [Figs. 6(a) and 6(b)] and an antimeron [Figs. 6(c) and 6(d)], at a tip-to-film working distance of $20 \mathrm{~nm}$, which is typical for this technique. The tip was modeled as a pyramid with dimensions $31 \times 31 \mathrm{~nm}^{2}$ base $\times 31 \mathrm{~nm}$ height, and a total magnetic moment of $1.2 \times 10^{-19} \mathrm{~J} \mathrm{~T}^{-1}$. Images were produced
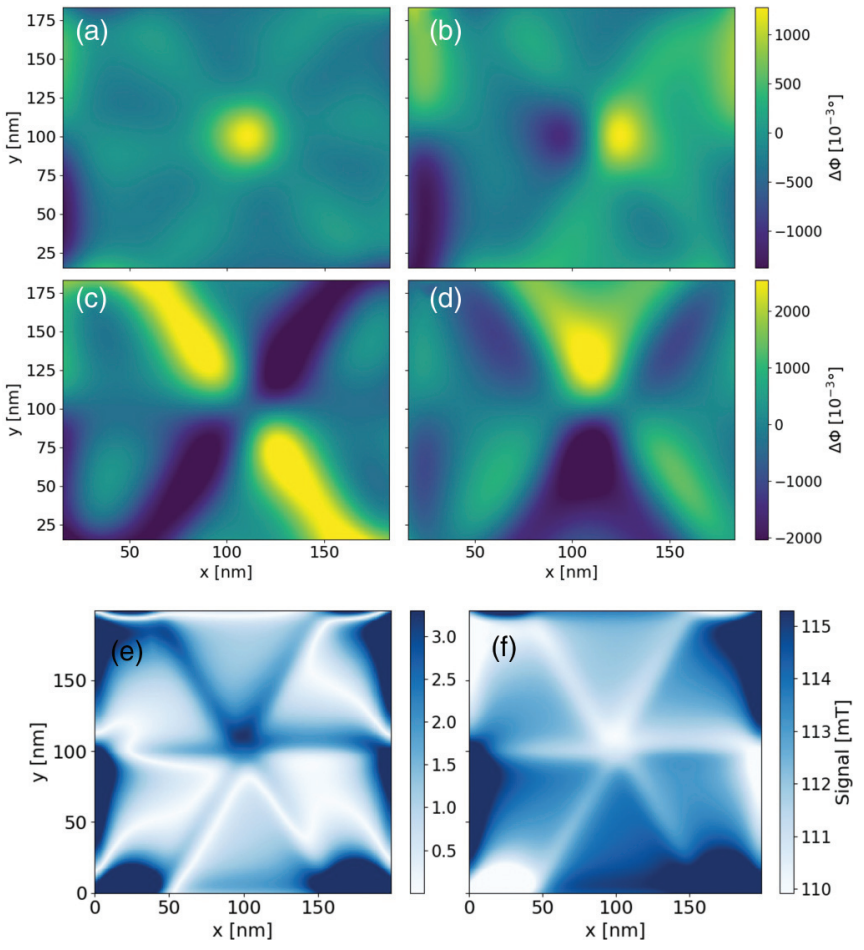

FIG. 6. Simulated MFM images of merons (top) and antimerons (middle) with both $L_{e x . b}$ and $L_{\text {ani }}$ parameters set to $3.8 \mathrm{~nm}$. In all cases, the magnetic moment of the tip was $1.15 \times 10^{-22} \mathrm{~J} \mathrm{~T}^{-1}$ and the working distance was $20 \mathrm{~nm}$. (a, c) Tip magnetized out of the page; $(\mathrm{b}, \mathrm{d})$ tip magnetized in the $y$ direction. Bottom: Images produced using an NV center tip with the $x$ axis of the sample aligned parallel to the in-plane projection of the defect, at a working distance of $11 \mathrm{~nm}$ : (e) no bias magnetic field and (f) bias field of $\sim 110 \mathrm{mT}$ along the $x$ direction.

with both perpendicular [Figs. 6(a) and 6(c)] and in-plane [Figs. 6(b) and 6(d)] magnetization of the tip. The meron core is distinguishable within typical instrumental sensitivity, albeit significantly broadened by resolution effects. With the tip magnetization perpendicular to the film [Fig. 6(a)], the core appears as a disk-shaped area of phase shift, and could be confused with other MFM features of different origin. By contrast, when the tip is magnetized in plane, the core displays a typical region of phase inversion [Fig. 6(b)], which could be used as a characteristic signature. Somewhat surprisingly, for antimerons [Figs. 6(c and 6(d)], the X-shaped ridge structure in the stray field is a much more prominent and recognizable feature than the core for both perpendicular and parallel tip magnetization.

Figures 6(e) and 6(f) show simulated NV center microscopy images of a meron, taken without and with a bias field in the direction of the defect axis, respectively. The working distance between the surface and the NV center was $11 \mathrm{~nm}$, which is realistic for a shallow defect. Because this technique is directly sensitive to the amplitude of the stray field, edge effects representing an artifact of the $200 \times 200 \mathrm{~nm}^{2}$ simulation region are very prominent in the simulated images. Nevertheless, details of the meron structure are very evident and are much less broadened by resolution effects than for MFM. In addition to the tight meron core, one can also 
clearly distinguish the Néel domain walls, which were all but invisible in MFM. Both unbiased and field-biased images are useful and provide complementary information, which can help unravel the magnetic structure of the meron. The NV center microscopy technique seems therefore very promising as an alternative and complement to X-PEEM, which has thus far been used exclusively to image these structures.

\section{CONCLUSIONS}

In conclusion, we have produced micromagnetic models and simulated MFM and NV center microscopy images of coupled (anti)vortex $\mid$ (anti)meron structures in $\alpha-\mathrm{Fe}_{2} \mathrm{O}_{3} \mid \mathrm{Co}$ heterostructures. Perhaps the most important conclusion of our analysis is that meron and antimeron cores in Co remain small $(<10 \mathrm{~nm})$ even when the exchange-bias interaction between AFM and FM layers is extremely weak. The fundamental reason for this is that the crossover between surface and bulk phenomenology (at strong and weak exchangebias interactions, respectively) is controlled by two different length scales, and that the bulk-related magnetostatic length keeps the FM features small even when the surface-related exchange-bias length is long. One outcome of this is that $\alpha-\mathrm{Fe}_{2} \mathrm{O}_{3} \mid \mathrm{Co}$ heterostructures and similar systems could, in principle, support very dense topological networks even in the presence of rough interfaces, which tend to weaken the net exchange-bias interaction. This is of course precisely what is wanted for applications, for example, in high-density magnetic storage.

One obstacle to fast-track development of these systems is the requirement for scarce X-PEEM beamtime at synchrotron sources to characterize the AFM and FM topological structures. Our simulated MFM and NV center microscopy images demonstrate the existence of characteristic features associated with FM merons and antimerons, which could be used to complement X-PEEM with much more accessible, laboratory-based techniques.

\section{ACKNOWLEDGMENTS}

Work done at the University of Oxford is funded by EPSRC Grant No. EP/M020517/1, entitled Oxford Quantum Materials Platform Grant. R.D.J. acknowledges support from a Royal Society University Research Fellowship. We thank S. Parameswaran for discussions and Tom Lancaster (University of Durham) and Hariom K. Jani (National University of Singapore) for commenting on the manuscript.

\section{APPENDIX A: EXCHANGE-BIAS PARAMETER AND MICROMAGNETIC SCALING}

In the exchange-bias calculations described in Sec. II B, we have employed the parameter $K_{\text {ex.b }}$ together with the definition of the exchange-bias energy:

$$
E_{\text {ex.b }}=\int d \sigma K_{\text {ex. } b}(1-\cos \theta),
$$

where $\theta$ is the angle between the AFM and the FM spins at the interface. Although in the OOMMF micromagnetic implementation it is not possible to introduce the equivalent of

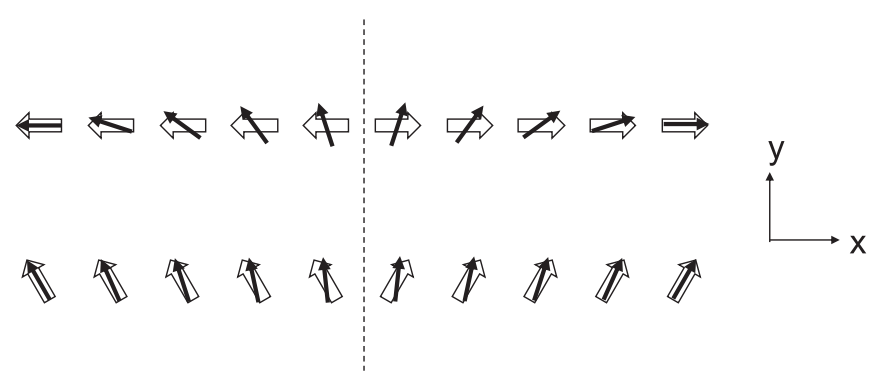

FIG. 7. Linear domain boundaries in Co (black arrows) induced by a sharp domain boundary in $\alpha-\mathrm{Fe}_{2} \mathrm{O}_{3}$ (white arrows representing the top uncompensated layer). Top: $180^{\circ}$ domain boundary. Bottom: $60^{\circ}$ domain boundary.

$K_{\text {ex.b }}$ directly, its effect can be reproduced by employing an exchange-stiffness parameter $A_{e x}^{\mathrm{Fe}-\mathrm{Co}}$, which acts only on the interface cells between $\alpha-\mathrm{Fe}_{2} \mathrm{O}_{3}$ and Co. In order to obtain a correct scaling of the model, one must be able to relate $A_{e x}^{\mathrm{Fe}-\mathrm{Co}}$ (which, as we see, is scale dependent) with the "physical" parameter $K_{\text {ex.b. }}$.

If at the interface the angle between the spins in $\alpha-\mathrm{Fe}_{2} \mathrm{O}_{3}$ and those in Co is $\theta$, the discrete gradient term is

$$
(\nabla)^{2}=\frac{1}{D_{z}^{2}}\left[(\cos \theta-1)^{2}+(\sin \theta)^{2}\right]=\frac{2}{D_{z}^{2}}(1-\cos \theta),
$$

where $D_{z}$ is the length of the discretization cell along the $z$ axis. The energy per unit area is therefore

$$
\begin{aligned}
\frac{\partial E_{e x . b}}{\partial \sigma} & =2 A_{e x}^{\mathrm{Fe}-\mathrm{Co}} \frac{2}{D_{z}^{2}}(1-\cos \theta) \frac{D_{z} D_{x y}^{2}}{D_{x y}^{2}} \\
& =2 \frac{A_{e x}^{\mathrm{Fe}-\mathrm{Co}}}{D_{z}}(1-\cos \theta),
\end{aligned}
$$

where $D_{x y}$ is the length of the discretization cell in the plane of the film. This is identical to the expression in Eq. (A1) [see also Eq. (B5)] with the identification $K_{e x . b}=2 A_{e x}^{\mathrm{Fe}-\mathrm{Co}} / D_{z}$. By performing simulations with different discretization cell sizes, we have verified that this is indeed the correct scaling factor to be applied for obtaining the same feature sizes in simulations with different $D_{z}$.

Expressions such as Eqs. (3) and (6) would also enable a value for $K_{e x . b}$ to be estimated from the feature sizes of experimental images, assuming that they are not limited by instrumental resolution.

\section{APPENDIX B: EXCHANGE-BIAS DOMAIN WALLS}

Here, we derive the width $W_{N}$ of a Néel domain wall induced in the Co overlayer by the exchange-bias interaction in the presence of a sharp $180^{\circ}$ antiphase AFM domain boundary in the $\alpha-\mathrm{Fe}_{2} \mathrm{O}_{3}$ film, and compare this result with the well-known, analogous calculation for the width $W_{B}$ of a Bloch wall in the presence of magnetocrystalline anisotropy. As discussed in the main text, we assume that the Co magnetization rotates by $180^{\circ}$ at a constant rate throughout the domain wall [Fig. 7(a)].

As a first step, we also assume the spin in Co to be coaligned along the $z$ axis (perpendicular to the film surface). 
Assuming the AFM spins in $\alpha-\mathrm{Fe}_{2} \mathrm{O}_{3}$ to be aligned along $\pm x$, the magnetization in the domain wall is described as

$$
M_{x}=M \cos \theta, \quad M_{y}=M \sin \theta,
$$

with

$$
\theta=\pi\left(\frac{x}{W_{N}}\right),
$$

$W_{N}$ being the full width of the domain wall in the $x$ direction.

The nonzero components of the gradient of the normalized magnetization gradients in Co are

$$
\frac{\partial m_{x}}{\partial x}=-\pi \frac{\sin \theta}{W_{N}}, \quad \frac{\partial m_{y}}{\partial x}=\pi \frac{\cos \theta}{W_{N}} .
$$

The exchange energy is therefore

$$
\begin{aligned}
E_{e x} & =A_{e x} \int_{0}^{W_{N}}\left(\frac{\partial m_{x}}{\partial x}\right)^{2}+\left(\frac{\partial m_{y}}{\partial x}\right)^{2} d v \\
& =A_{e x} \pi^{2} \frac{\mathcal{A}}{W_{N}},
\end{aligned}
$$

where $\mathcal{A}=d L$ is the area of the domain wall. This expression is identical to the exchange energy for a Bloch domain wall in the bulk.

We now calculate the exchange-bias energy, subtracting the FM energy as usual. This results from the following integral over the area:

$$
E_{e x . b}=\int d \sigma K_{\text {ex. }}(1-\cos \theta),
$$

where $K_{e x . b}$ is an energy per unit area. Performing the integral explicitly,

$$
\begin{aligned}
E_{\text {ex.b }} & =K_{\text {ex.b }} L 2 \int_{0}^{W_{N} / 2} d x\left(1-\cos \pi \frac{x}{W_{N}}\right) \\
& =K_{\text {ex. } b} W_{N} L\left(1-\frac{2}{\pi}\right) .
\end{aligned}
$$

Once again, this expression is very similar to the magnetocrystalline anisotropy energy for a Bloch domain wall, with the caveat that $K_{e x . b}$ is an energy per unit area, while $K_{a n}$ is an energy per unit volume:

$$
\begin{aligned}
E_{a n} & =K_{a n} L d \int_{0}^{W_{B}} d x \cos ^{2} \pi \frac{x}{W_{B}} \\
& =\frac{1}{2} K_{a n} W_{B} L d .
\end{aligned}
$$

By minimizing the total energy versus the width of the domain walls, one can easily find

$$
\begin{aligned}
& W_{N}=\pi\left(1-\frac{2}{\pi}\right)^{-1 / 2} \sqrt{\frac{A_{e x} d}{K_{e x . b}}} \approx 5.52 L_{e x . b}, \\
& W_{B}=\pi \sqrt{2} \sqrt{\frac{A_{e x}}{K_{a n}}} \approx 4.44 L_{a n},
\end{aligned}
$$

which is consistent with the discussion in Sec. II and the definition of the "exchange-bias length in Eq. (1).
Relaxing the assumption that the spin in Co is co-aligned along the $z$ axis, one can let the width of the domain wall depend on $z$, such that

$$
W_{N}(z)=W_{N}^{0}+\lambda z+\cdots,
$$

where the $z$ axis originates at the interface and $\lambda$ is a parameter to be determined by minimizing the total energy. This problem is slightly more complex but is tractable analytically. To first order, one finds that the Co spins remain strictly co-aligned (i.e., $\lambda=0$ ) unless $\frac{d}{L_{\text {ex } x}} \approx 1$, which is the case for the 8 -nm Co film discussed in Sec. IV B.

For the purpose of comparing with our simulations, it is also useful to calculate the width of a $60^{\circ}$ domain wall [Fig. 7(b)], which is defined by Eq. (B1) together with

$$
\theta=\frac{\pi}{3}\left(\frac{x}{W_{N}}+1\right)
$$

A calculation very similar to Eq. (B8) yields

$$
W_{N}^{60^{\circ}}=\frac{\pi}{3}\left(1-\frac{3}{\pi}\right)^{-1 / 2} \sqrt{\frac{A_{e x} d}{K_{e x . b}}} \approx 4.93 L_{\text {ex. } .} .
$$

The full width at half maximum is

$$
\mathrm{FWHM}^{60^{\circ}}=\frac{6}{\pi} \arcsin \left(\frac{1}{4}\right) W_{N}^{60^{\circ}} \approx 2.38 L_{\text {ex. } b .} .
$$

\section{APPENDIX C: DETAILED CALCULATION FOR THE ANALYTICAL MERONS}

Our aim here is to calculate the exchange energy difference between a meron of radius $R$ and a flat vortex with $R \rightarrow 0$, which is expected to be negative, since spins in the meron are almost parallel near the core. We first discuss the simplest case of $\mathcal{F}(x)=x$ (the "projective" meron). From Eq. (4) we have

$$
\begin{aligned}
& m_{x}=-\frac{r}{\sqrt{r^{2}+R^{2}}} \sin \phi, \quad m_{y}=\frac{r}{\sqrt{r^{2}+R^{2}}} \cos \phi, \\
& m_{z}=\frac{R}{\sqrt{r^{2}+R^{2}}} .
\end{aligned}
$$

We also consider the "linear meron" case:

$m_{x}=-\sin \theta \sin \phi, \quad m_{y}=\sin \theta \cos \phi, \quad m_{z}=\cos \theta$,

where

$$
\theta= \begin{cases}\frac{\pi r}{2 R} & \text { for } r \leqslant R \\ \frac{\pi}{2} & \text { for } r>R\end{cases}
$$

Using the expression for the gradient in cylindrical coordinates we can easily calculate the exchange energy. For example, for the projective meron,

$$
\begin{aligned}
E_{e x} & =A_{e x} \int d v\left(\frac{\partial}{\partial r}\right)^{2}+\left(\frac{1}{r} \frac{\partial}{\partial \phi}\right)^{2} \\
& =2 \pi A_{e x} d \int r d r \frac{r^{2}+2 R^{2}}{\left(r^{2}+R^{2}\right)^{2}} \\
& =\left.\pi A_{e x} d\left(\ln \left(r^{2}+R^{2}\right)-\frac{R^{2}}{r^{2}+R^{2}}\right)\right|_{0} ^{\infty} \\
& =\pi A_{e x} d\left(2 \lim _{r \rightarrow \infty} \ln \left(\frac{r}{R}\right)+1\right) .
\end{aligned}
$$


The general expression

$$
E_{e x}=\pi A_{e x} d\left(2 \lim _{r \rightarrow \infty} \ln \left(\frac{r}{R}\right)+c\right)
$$

holds in a variety of situations; in particular, when $\mathcal{F}(x)=x^{n}$ is a positive power of $x$, one can show that $c=n$. Moreover, if $\mathcal{F}(x)$ is zero outside a radius $R$ and $R$ is the only length scale involved, then $E_{e x}$ must be independent on $R$ due to simple dimensional considerations.

From $E_{e x}$, we must subtract the energy of a planar vortex $(R=0)$, where it is convenient to replace the lower limit of integration with a small length $a$, which is sent to zero at the end of the calculation. The vortex energy integrated to infinity is

$$
E_{\mathrm{vortex}}^{\infty}=\pi A_{e x} d\left(2 \lim _{r \rightarrow \infty} \ln \left(\frac{r}{a}\right)\right)
$$

while the vortex energy integrated to a radius $R$ is

$$
E_{\mathrm{vortex}}^{R}=\pi A_{e x} d\left(2 \ln \left(\frac{R}{a}\right)\right) .
$$

By performing the subtraction, one obtains the following general formula for the pure exchange energy of the core:

$$
\Delta E_{e x}=\pi A_{e x} d\left(-2 \ln \left(\frac{R}{a}\right)+c\right),
$$

which is always negative for $R \gg a$, and

$$
\frac{\partial \Delta E_{e x}}{\partial R}=-2 \pi A_{e x} d \frac{1}{R} .
$$

We now need to calculate the loss of exchange-bias energy occurring at the interface with respect to the vortex, due to the out-of-plane canting, which is obtained by performing the surface integral in Eq. (B5). With a straightforward calcula- tion one obtains for the projective meron $(\mathcal{F}(x)=x)$

$$
\Delta E_{\text {ex.b }}=\frac{\pi}{2} K_{\text {ex.b }} R^{2}\left(\ln \frac{4 r^{2}}{R^{2}}-1\right),
$$

which has a logarithmic divergence, due to the fact that the $m_{z}$ does not decay fast enough away from the core, while for the quadratic meron $\left(\mathcal{F}(x)=x^{2}\right)$,

$$
\Delta E_{\text {ex.b }}=\pi K_{\text {ex. } .} R^{2}
$$

and

$$
\frac{\partial \Delta E_{e x . b}}{\partial R}=2 \pi K_{e x . b} R .
$$

For the linear meron, the equivalent expressions are

$$
\Delta E_{\text {ex. } . b}=2 \pi K_{\text {ex.b }} \int_{0}^{R} r \sin \left(\frac{\pi r}{2 R}\right) d r=\frac{8}{\pi} K_{\text {ex. } . b} R^{2}
$$

and

$$
\frac{\partial \Delta E_{e x . b}}{\partial R}=2 \pi \frac{8}{\pi^{2}} K_{e x . b} R .
$$

We need to minimize the expression

$$
\Delta E_{t o t}=\Delta E_{e x . b}+\Delta E_{e x}
$$

as a function of $R$, which is easily done with the help of Eqs. (C9), (C12), and (C14), yielding

$$
R_{\min }=\kappa \sqrt{\frac{A_{e x} d}{K_{e x . b}}}=\kappa L_{e x . b},
$$

where $\kappa=1$ for the quadratic meron and $\kappa=\pi \sqrt{2} / 4 \approx 1.11$ for the linear meron.

The linear meron can be directly compared with the linear Néel domain wall by observing that the lengths over which the spins rotate by $180^{\circ}$ are $W_{N} \approx 5.52 L_{\text {ex.b }}$ [Eq. (B8)] and $W_{\text {core }}=2 R_{\min } \approx 2.22 L_{\text {ex.b }}$ [Eq. (C16)]. Another useful quantity is the FWHM of the $M_{z}$ peak, which is directly comparable to our simulations. A very simple analysis yields

$$
\mathrm{FWHM}=\frac{2}{3} W_{\text {core }} \approx 1.5 L_{\text {ex. } b} .
$$

[1] S. Manipatruni, D. E. Nikonov, and I. A. Young, Nat. Phys. 14, 338 (2018).

[2] W. Jiang, G. Chen, K. Liu, J. Zang, S. G. te Velthuis, and A. Hoffmann, Phys. Rep. 704, 1 (2017).

[3] T. Lancaster, Contemp. Phys. 60, 246 (2019).

[4] T. Shinjo, T. Okuno, R. Hassdorf, K. Shigeto, and T. Ono, Science 289, 930 (2000).

[5] X. Z. Yu, W. Koshibae, Y. Tokunaga, K. Shibata, Y. Taguchi, N. Nagaosa, and Y. Tokura, Nature (London) 564, 95 (2018).

[6] T. Eggebrecht, M. Möller, J. G. Gatzmann, N. Rubiano da Silva, A. Feist, U. Martens, H. Ulrichs, M. Münzenberg, C. Ropers, and S. Schäfer, Phys. Rev. Lett. 118, 097203 (2017).

[7] F. P. Chmiel, N. Waterfield Price, R. D. Johnson, A. D. Lamirand, J. Schad, G. van der Laan, D. T. Harris, J. Irwin, M. S. Rzchowski, C.-B. Eom, and P. G. Radaelli, Nat. Mater. 17, 581 (2018).
[8] C. G. Callan, R. Dashen, and D. J. Gross, Phys. Rev. D 17, 2717 (1978).

[9] Strictly speaking, a spin-up meron can be converted to a spindown antimeron by a global rotation in spin space, but this requires to rotate a large number of spins by wide angles.

[10] F. Nolting, A. Scholl, J. Stöhr, J. W. Seo, J. Fompeyrine, H. Siegwart, J.-P. Locquet, S. Anders, J. Lüning, E. E. Fullerton, M. F. Toney, M. R. Scheinfein, and H. A. Padmore, Nature (London) 405, 767 (2000).

[11] A. Papp, W. Porod, and G. Csaba, J. Appl. Phys. 117, 17E101 (2015).

[12] E. G. Galkina, A. Y. Galkin, B. A. Ivanov, and F. Nori, Phys. Rev. B 81, 184413 (2010).

[13] We have in fact obtained very recent experimental evidence of the existence of out-of-plane meron cores in pure $\alpha-\mathrm{Fe}_{2} \mathrm{O}_{3}$.

[14] A. Scholl, M. Liberati, E. Arenholz, H. Ohldag, and J. Stöhr, Phys. Rev. Lett. 92, 247201 (2004). 
[15] G. S. Abo, Y.-K. Hong, J. Park, J. Lee, W. Lee, and B.-C. Choi, IEEE Trans. Magn. 49, 4937 (2013).

[16] A. Aharoni, J. Appl. Phys. 37, 3271 (1966).

[17] The general form of the differential equation for a $180^{\circ}$ domain wall is $\Theta^{\prime \prime}(x)-\frac{s(x)}{L_{\text {ex. }}^{2}} \sin \Theta(x)=0$, where $\Theta(x)$ is the spin angle of the equilibrium structure at position $x, L_{e x . b}$ is the exchange-bias length, and $s(x)=\operatorname{sgn}(x)$ is the sign function. The second derivative of $\Theta(x)$ is clearly discontinuous at the origin, but a closed-form solution of this equation exists and can be expressed in terms of Jacobi functions. The linear domain wall is a reasonable approximation of these solutions.

[18] R. Rajaraman, Solitons and Instantons: An Introduction to Solitons and Instantons in Quantum Field Theory (North-Holland, Amsterdam, 1987), p. 409.

[19] M. Donahue and D. Porter, OOMMF User's Guide, Version 1.0 (NIST, Gaithersburg, MD, 1999).
[20] T. L. Gilbert, IEEE Trans. Magn. 40, 3443 (2004).

[21] U. Hartmann, Annu. Rev. Mater. Sci. 29, 53 (1999).

[22] L. Rondin, J.-P. Tetienne, T. Hingant, J.-F. Roch, P. Maletinsky, and V. Jacques, Rep. Prog. Phys. 77, 056503 (2014).

[23] I. Gross, W. Akhtar, V. Garcia, L. J. Martínez, S. Chouaieb, K. Garcia, C. Carrétéro, A. Barthélémy, P. Appel, P. Maletinsky, J.-V. Kim, J. Y. Chauleau, N. Jaouen, M. Viret, M. Bibes, S. Fusil, and V. Jacques, Nature (London) 549, 252 (2017).

[24] This is a natural generalization of the usual $180^{\circ}$ domain wall model to the case of a trigonal system having $60^{\circ}$ domains. It follows that, for a constant-magnetization $60^{\circ}$ domain wall, the perpendicular component is at most $1 / 2$ of the total magnetization.

[25] Quantum Magnetism, edited by U. Schollwöck, J. Richter, D. J. J. Farnell, and R. F. Bishop, Lecture Notes in Physics Vol. 645 (Springer, Berlin, 2004). 